\title{
VSEARCH: a versatile open source tool for metagenomics
}

\author{
Torbjørn Rognes ${ }^{\text {Corresp., }}{ }^{1,2}{ }^{\text {， Tomáš Flouri }}{ }^{3,4}$, Ben Nichols ${ }^{5}$, Christopher Quince ${ }^{5,6}$, Frédéric Mahé ${ }^{7,8}$ \\ 1 Department of Informatics, University of Oslo, Oslo, Norway \\ 2 Department of Microbiology, Oslo University Hospital, Oslo, Norway \\ 3 Heidelberg Institute for Theoretical Studies, Heidelberg, Germany \\ 4 Institute for Theoretical Informatics, Karlsruhe Institute of Technology, Karlsruhe, Germany \\ 5 School of Engineering, University of Glasgow, Glasgow, United Kingdom \\ 6 Warwick Medical School, University of Warwick, Coventry, United Kingdom \\ 7 Department of Ecology, University of Kaiserslautern, Kaiserslautern, Germany \\ 8 UMR LSTM, CIRAD, Montpellier, France \\ Corresponding Author: Torbjørn Rognes \\ Email address: torognes@ifi.uio.no
}

Background. VSEARCH is an open source and free of charge multithreaded 64-bit tool for processing metagenomics nucleotide sequence data. It is designed as an alternative to the widely used USEARCH tool (Edgar 2010) for which the source code is not publicly available, algorithm details are only rudimentarily described, and only a memory-confined 32-bit version is freely available for academic use.

Methods. When searching nucleotide sequences, VSEARCH uses a fast heuristic based on words shared by the query and target sequences in order to quickly identify similar sequences, a similar strategy is probably used in USEARCH. VSEARCH then performs optimal global sequence alignment of the query against potential target sequences, using full dynamic programming instead of the seed-and-extend heuristic used by USEARCH. Pairwise alignments are computed in parallel using vectorisation and multiple threads.

Results. VSEARCH includes most commands for analysing nucleotide sequences available in USEARCH version 7 and several of those available in USEARCH version 8, including searching (exact or based on global alignment), clustering by similarity (using length pre-sorting, abundance pre-sorting or a userdefined order), chimera detection (reference-based or de novo), dereplication (full length or prefix), pairwise alignment, reverse complementation, sorting, and subsampling. VSEARCH also includes commands for FASTQ file processing, i.e. format detection, filtering, read quality statistics, and merging of paired reads. Furthermore, VSEARCH extends functionality with several new commands and improvements, including shuffling, rereplication, masking of low-complexity sequences with the wellknown DUST algorithm, a choice among different similarity definitions, and FASTQ file format conversion. VSEARCH is here shown to be more accurate than USEARCH when performing searching, clustering, chimera detection and subsampling, while on a par with USEARCH for paired-ends read merging. VSEARCH is slower than USEARCH when performing clustering and chimera detection, but significantly faster when performing paired-end reads merging and dereplication. VSEARCH is available at https://github.com/torognes/vsearch under either the BSD 2-clause license or the GNU General Public License version 3.0.

Discussion. VSEARCH has been shown to be a fast, accurate and full-fledged alternative to USEARCH. A free and open-source versatile tool for sequence analysis is now available to the metagenomics community. 


\section{VSEARCH: a versatile open source tool for 2 metagenomics}

3

4 Short title:

5 VSEARCH: a versatile metagenomics tool 6

$9{ }^{1}$ Department of Informatics, University of Oslo, Oslo, Norway

$10{ }^{2}$ Department of Microbiology, Oslo University Hospital, Rikshospitalet, Oslo, Norway

$11{ }^{3}$ Heidelberg Institute for Theoretical Studies (HITS), Heidelberg, Germany

$12{ }^{4}$ Karlsruhe Institute of Technology, Institute for Theoretical Informatics, Karlsruhe, Germany

$13{ }^{5}$ School of Engineering, University of Glasgow, Glasgow, UK

$14{ }^{6}$ Warwick Medical School, University of Warwick, Coventry, UK

$15{ }^{7}$ Department of Ecology, University of Kaiserslautern, Germany

$16{ }^{8}$ CIRAD, UMR LSTM, Montpellier, France

17
* Corresponding author. Address: Department of Informatics, University of Oslo, PO Box 1080 Blindern, NO-0316 Oslo, Norway. 


\section{Abstract}

28

Background. VSEARCH is an open source and free of charge multithreaded 64-bit tool for processing metagenomics nucleotide sequence data. It is designed as an alternative to the widely used USEARCH tool (Edgar 2010) for which the source code is not publicly available, algorithm details are only rudimentarily described, and only a memory-confined 32-bit version is freely available for academic use.

Methods. When searching nucleotide sequences, VSEARCH uses a fast heuristic based on words shared by the query and target sequences in order to quickly identify similar sequences, a similar strategy is probably used in USEARCH. VSEARCH then performs optimal global sequence alignment of the query against potential target sequences, using full dynamic programming instead of the seed-and-extend heuristic used by USEARCH. Pairwise alignments are computed in parallel using vectorisation and multiple threads.

Results. VSEARCH includes most commands for analysing nucleotide sequences available in USEARCH version 7 and several of those available in USEARCH version 8, including searching (exact or based on global alignment), clustering by similarity (using length pre-sorting, abundance pre-sorting or a user-defined order), chimera detection (reference-based or de novo), dereplication (full length or prefix), pairwise alignment, reverse complementation, sorting, and subsampling. VSEARCH also includes commands for FASTQ file processing, i.e. format detection, filtering, read quality statistics, and merging of paired reads. Furthermore, VSEARCH extends functionality with several new commands and improvements, including shuffling, rereplication, masking of low-complexity sequences with the well-known DUST algorithm, a choice among different similarity definitions, and FASTQ file format conversion. VSEARCH is here shown to be more accurate than USEARCH when performing searching, clustering, chimera detection and subsampling, while on a par with USEARCH for paired-ends read merging. VSEARCH is slower than USEARCH when performing clustering and chimera detection, but significantly faster when performing paired-end reads merging and dereplication. VSEARCH is available at https://github.com/torognes/vsearch under either the BSD 2-clause license or the GNU General Public License version 3.0.

Discussion. VSEARCH has been shown to be a fast, accurate and full-fledged alternative to USEARCH. A free and open-source versatile tool for sequence analysis is now available to the metagenomics community. 


\section{Subjects}

63 Biodiversity, Bioinformatics, Computational Biology, Genomics, Microbiology

\section{Keywords}

alignment, clustering, chimera detection, dereplication, metagenomics, searching, sequences, masking, shuffling, parallelization

\section{Introduction}

Rockström et al. (2009) and Steffen et al. (2015) presented biodiversity loss as a major threat for the short-term survival of humanity. Recent progress in sequencing technologies have made possible large scale studies of environmental genetic diversity, from deep sea hydrothermal vents to Antarctic lakes (Karsenti et al., 2011), and from tropical forests to Siberian steppes (Gilbert, Jansson and Knight, 2014). Recent clinical studies have shown the importance of the microbiomes of our bodies and daily environments for human health (Human Microbiome Project Consortium, 2012). Usually focusing on universal markers (e.g., 16S rRNA, ITS, COI), these targeted metagenomics studies produce many millions of sequences, and require opensource, fast and memory efficient tools to facilitate their ecological interpretation.

Several pipelines have been developed for microbiome analysis, among which mothur (Schloss et al., 2009), QIIME (Caporaso et al., 2010), and UPARSE (Edgar, 2013) are the most popular. QIIME and UPARSE are both based on USEARCH (Edgar, 2010), a set of tools designed and implemented by Robert C. Edgar, and available at http://drive5.com/usearch/. USEARCH offers a great number of commands and options to manipulate and analyse FASTQ and FASTA files. However, the source code of USEARCH is not publicly available, algorithm details are only rudimentarily described, and only a memory-confined 32-bit version is freely available for academic use.

We believe that the existence of open-source solutions is beneficial for end-users and can invigorate research activities. For this reason, we have undertaken to offer a high quality opensource alternative to USEARCH, freely available to users without any memory limitation. VSEARCH includes most of the USEARCH functions in common use, and further development may add additional features. Here we describe the details of the VSEARCH implementation. To assess its performance in terms of speed and quality of results, we have evaluated some of the most important functions (searching, clustering, chimera detection and subsampling) and compared them to USEARCH. We find that VSEARCH delivers results that are better or on a

97 par with USEARCH results. 
Materials and Methods

99

100

101

102

103

104

105

106

107

108

109

110

111

112

113

114

115

116

117

118

119

120

121

122

123

124

125

126

127

128

129

130

131

132

133

134

135

136

\section{Algorithms and implementation}

Below is a brief description of the most important functions of VSEARCH and details of their implementation. VSEARCH command line options are shown in italics, and should be preceded by a single (-) or double dash (--) when used.

\section{Reading FASTA and FASTQ files}

Most VSEARCH commands read files in FASTA or FASTQ format. The parser for FASTQ files in VSEARCH is compliant with the standard as described by Cock et al. (2010) and correctly parses all their tests files. FASTA and FASTQ files are automatically detected and many commands accept both as input. Files compressed with gzip or bzip2 are automatically detected and decompressed using the zlib library by Gailly and Adler (2016) or the bzip2 library by Seward (2016), respectively. Input may also be piped into or out of VSEARCH, allowing for instance many separate FASTA files to be piped into VSEARCH for simultaneous dereplication, or allowing the creation of complex pipelines without ever having to write on slow disks.

VSEARCH is a 64-bit program and allows very large datasets to be processed, essentially limited only by the amount of memory available. The free USEARCH versions are 32-bit programs that limit the available memory to somewhere less than 4GB, often seriously hampering the analysis of realistic datasets.

\section{Writing result files}

VSEARCH can output results in a variety of formats (FASTA, FASTQ, tables, alignments, SAM) depending on the input format and command used. When outputting FASTA files, the line width may be specified using the fasta_width option, where 0 means that line wrapping should be turned off. Similar controls are offered for pairwise or multiple sequence alignments.

\section{Searching}

Global pairwise sequence comparison is a core-functionality of VSEARCH. Several commands compare a query sequence against a database of sequences: all-vs-all alignment (allpairs_global), clustering (cluster_fast, cluster_size, cluster_smallmem), chimera detection (uchime_denovo and uchime_ref) and searching (usearch_global). This comparison function proceeds in two phases: an initial heuristic filtering based on shared words, followed by optimal alignment of the query with the most promising candidates.

The first phase is presumably quite similar to USEARCH (Edgar, 2010). Heuristics are used to identify a small set of database sequences that have many words in common with the query sequence. Words (or $k$-mers) consist of a certain number $k$ of consecutive nucleotides of a sequence ( 8 by default, adjustable with the wordlength option). All overlapping words are 
137 included. A sequence of length $n$ then contains at most $n-k+1$ unique words. VSEARCH

138 counts the number of shared words between the query and each database sequence. Words that

139 appear multiple times are counted only once. To count the words in the database sequences

140 quickly, VSEARCH creates an index of all the $4^{k}$ possible distinct words and stores information

141 about which database sequences they appear in. For extremely frequent words, the set of

142 database sequences is represented by a bitmap; otherwise the set is stored as a list. A finer

143 control of $k$-mer indexing is possible by introducing the pattern (binary string indicating which

144 positions must match) and slots options. USEARCH has such options but seems to ignore them.

145 Currently, VSEARCH ignores these two options too. The minimum number of shared words

146 required may be specified with the minwordmatches option (10 by default), but a lower value is

147 automatically used for short or simple query sequences with less than 10 unique words.

148

149 Comparing sequences based on statistics of shared words is a common method to quickly assess

150 the similarity between two sequences without aligning them, which is often time-consuming. The

$151 D_{2}$ statistic and related metrics for alignment-free sequence comparison have often been used for

152 rapid and approximate sequence matching and their statistical properties have been well studied

153 (Song et al., 2014). The approach used here has similarities to the $D_{2}$ statistic, but multiple

154 matches of the same word are ignored.

155

156 In the second phase, searching proceeds by considering the database sequences in a specific

157 order, starting with the sequence having the largest number of words in common with the query,

158 and proceeding with a decreasing number of shared words. If two database sequences have the

159 same number of words in common with the query, the shortest sequence is considered first. The

160 query sequence is compared with each database sequence by computing the optimal global

161 alignment. The alignment is performed using a multi-threaded and vectorised full dynamic

162 programming algorithm (Needleman and Wunsch, 1970) adapted from SWIPE (Rognes, 2011).

163 Due to the extreme memory requirements of this method when aligning two long sequences, an

164 alternative algorithm described by Hirschberg (1975) and Myers and Miller (1988) is used when

165 the product of the length of the sequences is greater than 25,000,000, corresponding to aligning

166 two 5,000 bp sequences. This alternative algorithm uses only a linear amount of memory but is

167 considerably slower. This second phase is probably where USEARCH and VSEARCH differ the

168 most, as USEARCH by default presumably performs a heuristic seed-and-extend alignment

169 similar to BLAST (Altschul et al., 1990), and only performs optimal pairwise alignments when

170 the option fulldp (full dynamic programming) is used. Computing the optimal pairwise alignment

171 in each case gives more accurate results but is also computationally more demanding. The

172 efficient and vectorised full dynamic programming implementation in VSEARCH compensates

173 that extra cost, at least for sequences that are not too long.

174

175 If the resulting alignment indicates a similarity equal to or greater than the value specified with

176 the id option, the database sequence is accepted. If the similarity is too low, it is rejected. Several 
177 other options may also be used to determine how similarity is computed (iddef, as USEARCH

178 used to offer up to version 6), and which sequences should be accepted and rejected, either

179 before (e.g. self, minqsize) or after alignment (e.g. maxgaps, maxsubs). The search is terminated

180 when either a certain number of sequences have been accepted ( 1 by default, adjustable with the

181 maxaccepts option), or a certain number of sequences have been rejected ( 32 by default,

182 adjustable with the maxrejects option). The accepted sequences are sorted by sequence similarity

183 and presented as the search results.

184

185 VSEARCH also includes a search_exact command that only identifies exact matches to the

186 query. It uses a hash table in a way similar to the full-length dereplication command described

187 below.

188

189

190

191

192

193

194

\section{Clustering}

VSEARCH includes commands to perform de novo clustering using a greedy and heuristic centroid-based algorithm with an adjustable sequence similarity threshold specified with the $i d$ option (e.g., 0.97). The input sequences are either processed in the user supplied order (cluster_smallmem) or pre-sorted based on length (cluster_fast) or abundance (the new cluster_size option). Each input sequence is then used as a query in a search against an initially empty database of centroid sequences. The query sequence is clustered with the first centroid sequence found with similarity equal to or above the threshold. The search is performed using the heuristic approach described above which generally finds the most similar sequences first. If no matches are found, the query sequence becomes the centroid of a new cluster and is added to the database. If maxaccepts is higher than 1 , several centroids with sufficient sequence similarity may be found and considered. By default, the query is clustered with the centroid presenting the highest sequence similarity (distance-based greedy clustering, DGC), or, if the sizeorder option is turned on, the centroid with the highest abundance (abundance-based greedy clustering, AGC) (He et al., 2015; Westcott and Schloss, 2015; Schloss, 2016). VSEARCH performs multithreaded clustering by searching the database of centroid sequences with several query sequences in parallel. If there are any non-matching query sequences giving rise to new centroids, the required internal comparisons between the query sequences are subsequently performed to achieve correct results. For each cluster, VSEARCH can perform a simple center-star multiple sequence alignment to compute consensus sequences and sequence profiles.

\section{Dereplication and rereplication}

Full-length dereplication (derep_fulllength) is performed using a hash table with an open addressing and linear probing strategy based on the Google CityHash hash functions (written by Geoff Pike and Jyrki Alakuijala, and available at https://github.com/google/cityhash). The hash table is initially empty. For each input sequence, the hash is computed and a lookup in the hash table is performed. If an identical sequence is found, the input sequence is clustered with the matching sequence; otherwise the input sequence is inserted into the hash table. 
218 Prefix dereplication (derep_prefix) is also implemented. As with full-length dereplication, 219 identical sequences are clustered. In addition, sequences that are identical to prefixes of other 220 sequences will also be clustered together. If a sequence is identical to the prefix of multiple 221 sequences, it is generally not defined how prefix clustering should behave. VSEARCH resolves 222 this ambiguity by clustering the sequence with the shortest of the candidate sequences. If they are 223 equally long, priority will be given to the most abundant, the one with the lexicographically 224 smaller identifier or the one with the earliest original position, in that order.

225

226

227

228

229

230

231

232

233

234

235

236

237

238

239

240

241

242

243

244

245

246

247

248

249

250

251

252

253

254

255

To perform prefix dereplication, VSEARCH first creates an initially empty hash table. It then sorts the input sequences by length and identifies the length $s$ of the shortest sequence in the dataset. Each input sequence is then processed as follows, starting with the shortest: If an exact match to the full input sequence is found in the hash table, the input sequence is clustered with the matching hash table sequence. If no match to the full input sequence is found, the prefixes of the input sequence are considered, starting with the longest prefix and proceeding with shorter prefixes in order, down to prefixes of length $s$. If a match is now found in the hash table, the sequences are clustered, the matching sequence is deleted from the hash table and the full input sequence is inserted into the hash table instead. If no match is found for any prefix, the full sequence is inserted into the hash table. In the end, the remaining sequences in the hash table will be output with accumulated abundances for all sequences in each cluster.

In order to identify matches in the hash table during prefix dereplication, a hash is computed for each full-length input sequence and all its prefixes. The hash function used is the 64-bit FowlerNoll-Vo la hash function (Fowler et al., 1991), which is simple and quick to compute for such a series of sequences by adding one nucleotide at a time.

The sequences resulting from dereplication and many other commands may be relabeled with a given prefix followed by a sequentially increasing number. VSEARCH exclusively also offers the possibility of relabelling each sequence with the SHA-1 (Eastlake and Jones, 2001) or MD5 (Rivest, 1992) message digest (hash) of the sequence. These are strings that are highly likely to be unique for each sequence. Before the digest is computed, the sequence is normalized by converting U's to T's and converting all symbols to upper case. VSEARCH includes public domain code for the MD5 algorithm written by Alexander Peslyak, and for SHA1 by Steve Reid and others.

VSEARCH also includes a new command (rereplicate) to perform rereplication that can be used to recreate datasets has they were before full-length dereplication, but of course original labels cannot be recreated. 
256 Chimera detection

257 Chimeras are detected either de novo (uchime_denovo command) or with a reference database

258 (uchime_ref command) using the UCHIME algorithm described by Edgar et al. (2011).

259 VSEARCH will divide each query sequence into four segments and look for similarity of each

260 segment to sequences in the set of potential parents using the heuristic search function described

261 earlier. It will consider the four best candidates for each segment using maxaccepts 4 and

262 maxrejects 16 , and an id threshold of 0.55 . VSEARCH optionally outputs borderline sequences,

263 that is, sequences having a high enough score (as specified with the minh option) but with too

264 small a divergence from the closest parent (as specified with the mindiv option). Multi-threading

265 is supported for reference-based chimera detection.

266

267

\section{Low-complexity sequence masking}

268 VSEARCH includes a highly optimized and parallelized implementation of the Dust algorithm

269 by Tatusov and Lipman for masking of simple repeats and low-complexity nucleotide sequences,

270 that is considerably faster than the implementation of the same algorithm in USEARCH. Their

271 code available at ftp://ftp.ncbi.nlm.nih.gov/pub/tatusov/dust/version1/src/ is in the public

272 domain. VSEARCH uses this algorithm by default, while USEARCH by default uses an

273 undocumented rapid masking algorithm called fastnucleo. VSEARCH performs soft-masking

274 automatically for the pairwise alignment, search, clustering and chimera detection commands.

275 This behaviour can be controlled with the hardmask option to replace masked symbols with N's

276 instead of lower-casing them, and the dbmask and qmask options, which selects the masking

277 algorithm (none, dust or soft) used for the database and query sequences, respectively. Masking

278 may also be performed explicitly on an input file using the fastx_mask and maskfasta commands.

279

280

281

282

283

284

285

\section{FASTQ file processing}

VSEARCH includes commands to detect the FASTQ file version and the range of quality scores used (fastq_chars), as well as two commands for computing sequence quality statistics

(fastq_stats and fastq_eestats). It can also truncate and filter sequences in FASTQ files based on various criteria (fastq_filter). A new command is added to convert between different FASTQ file versions and quality encodings (fastq_convert), e.g. from the old Phred +64 encoded Illumina

286 FASTQ files to the newer Phred +33 format.

287

288

289

\section{Merging of paired-end reads}

Merging of paired-end reads is supported by VSEARCH using the fastq_mergepairs command.

290

291 The method used has some similarity to PEAR (Zhang et al., 2014) and recognises options similar to USEARCH. The algorithm computes the optimal ungapped alignment of the overlapping region of the forward sequence and the reverse-complemented reverse sequence.

293 The alignment requires a minimum overlap length (specified with the fastq_minovlen option,

294 default 10), a maximum number of mismatches (fastq_maxdiffs option, default 5), and a

295 minimum and maximum length of the merged sequence (fastq_minmergelen option, default 1, 
296

297

298

299

300

301

302

303

304

305

306

307

308

309

310

311

312

313

314

315

316

317

318

319

320

321

322

323

324

325

326

327

328

329

330

331

332

333

334

and fastq_maxmergelen option, default infinite). Staggered read pairs, i.e. read pairs where the 3' end of the reverse read has an overhang to the left of the 5' end of the forward read, are not allowed by default, but may be turned on by the fastq_allowmergestagger option. VSEARCH uses a match score (alpha) of +4 and a mismatch score (beta) of -5 for perfect quality residues. These scores are weighted by the probability that these two residues really match or mismatch, respectively, taking quality scores into account. These probabilities are computed in a way similar to PEAR score method 2 described in section 2.1 of the PEAR paper (Zhang et al., 2014), but VSEARCH assumes all nucleotide background frequencies are 0.25 . When merging sequences, VSEARCH computes posterior quality scores for the overlapping regions as described by Edgar and Flyvbjerg (2015). For speed, scores and probabilities are pre-computed for all possible quality scores.

\section{Sorting and shuffling}

VSEARCH can sort FASTA files by decreasing sequence length (sortbylength) or abundance (sortbysize). VSEARCH can also perform shuffling of FASTA files in random order (shuffle). A seed value for the pseudo random number generator may be provided by the randseed option to obtain replicable results.

\section{Subsampling}

Sequences in FASTA and FASTQ files can be subsampled (fastx_subsample) by randomly extracting a certain number (sample_size) or percentage (sample_pct) of the input sequences. Abundances may be taken into account, giving results as if the input sequences were rereplicated, subsampled and then dereplicated.

\section{Results and Discussion}

\section{Supported commands and options}

VSEARCH implements the following commands available in USEARCH version 7: allpairs_global, cluster_fast, cluster_smallmem, derep_fulllength, derep_prefix, fastq_chars, fastq_filter,fastq_mergepairs, fastq_stats,fastx_mask, maskfasta, sortbylength, sortbysize, uchime_denovo, uchime_ref and usearch_global. In addition, the following commands available in USEARCH version 8 have been implemented:fastq_eestats, fastx_revcomp, fastx_subsample and search_exact. VSEARCH additionally includes a few new commands that do not exist in USEARCH: cluster_size,fastq_convert, rereplicate and shuffle.

Some USEARCH version 7 commands have not yet been implemented in VSEARCH. We have not prioritized commands related to amino acid sequences (findorfs), local alignment (allpairs_local,pairs_local, search_local,ublast), brute-force search (search_global, pairs_global), UDB databases (makeudb_ublast, makeudb_usearch, udb2fasta, udbinfo, udbstats), and the UPARSE pipeline (cluster_otus, uparse_ref). 
336 Almost all USEARCH 7 options are supported, except for those related to non-standard database indexing (alpha, dbaccelpct, dbstep, pattern, slots) as well as local alignments and alignment heuristics (band, hspw, lext, lopen, matrix, minhsp, xdrop_g, xdrop_nw, xdrop_u).

339

The same command and option names as in USEARCH version 7 has generally been used in order to make VSEARCH an almost drop-in replacement. In fact, in QIIME most commands will run fine if an alias or link from usearch to vsearch is made. Detailed documentation of VSEARCH is available as a man page. We will consider adding further commands and options to VSEARCH in the future.

345

\section{Performance Assessment}

The performance of the most important functions of VSEARCH version 2.0.3 was evaluated and compared to USEARCH version 7.0.1090 and 8.1.1861. Chimera detection was also compared to UCHIME version 4.2. All tests were run on GNU/Linux CentOS 6.7 compute nodes with 16 physical cores (Intel(R) Xeon(R) CPU E5-2670 0 @ 2.60GHz) and 64GB RAM. Programs were run with 8 threads, if possible. All times indicated are wall-clock times. All scripts and data necessary to perform the evaluations are available in the GitHub repository at https://github.com/torognes/vsearch-eval/ to enable independent replication.

355

356

357

358

359

360

361

362

363

364

365

366

367

368

369

370

371

372

373

\section{Searching}

Evaluation of search accuracy was carried out as described in the USEARCH paper (Edgar, 2010), its supplementary, and on the website (http://drive5.com/usearch/benchmark_rfam.html), by assessing the ability of the programs to identify RNA sequences belonging to the same family in RFAM (Burge et al., 2013). The 383,004 sequences in Rfam version 11 were randomly shuffled and then the first sequence from each of the 2,085 (out of 2,208) families that contained at least 2 members was selected as a representative and used as a query against the remaining 380,919 sequences. The programs were run with options id 0.0 , minseqlength 1 , maxaccepts 1 , maxrejects 32 , and strand plus. If the matching sequence found belonged to the same family, it was considered a true positive, otherwise it was considered as a false positive. We combined the results from 20 shufflings and plotted the results in the ROC-like curve shown in Fig. 1. For a false discovery rate comprised between 0.010 and 0.015 , VSEARCH is more accurate than USEARCH's latest version. For lower values, the three programs have similar accuracies. At higher false discovery rates, USEARCH version 8 has an advantage.

The time to search the Rfam database as described above was measured. To avoid extremely short running times, 1,000 replicates of the datasets were used. USEARCH version 7 required on average $5 \mathrm{~min} 29$ seconds for the search, USEARCH version 8 took 5 min 57 seconds, while VSEARCH took 5 min 26 seconds. 


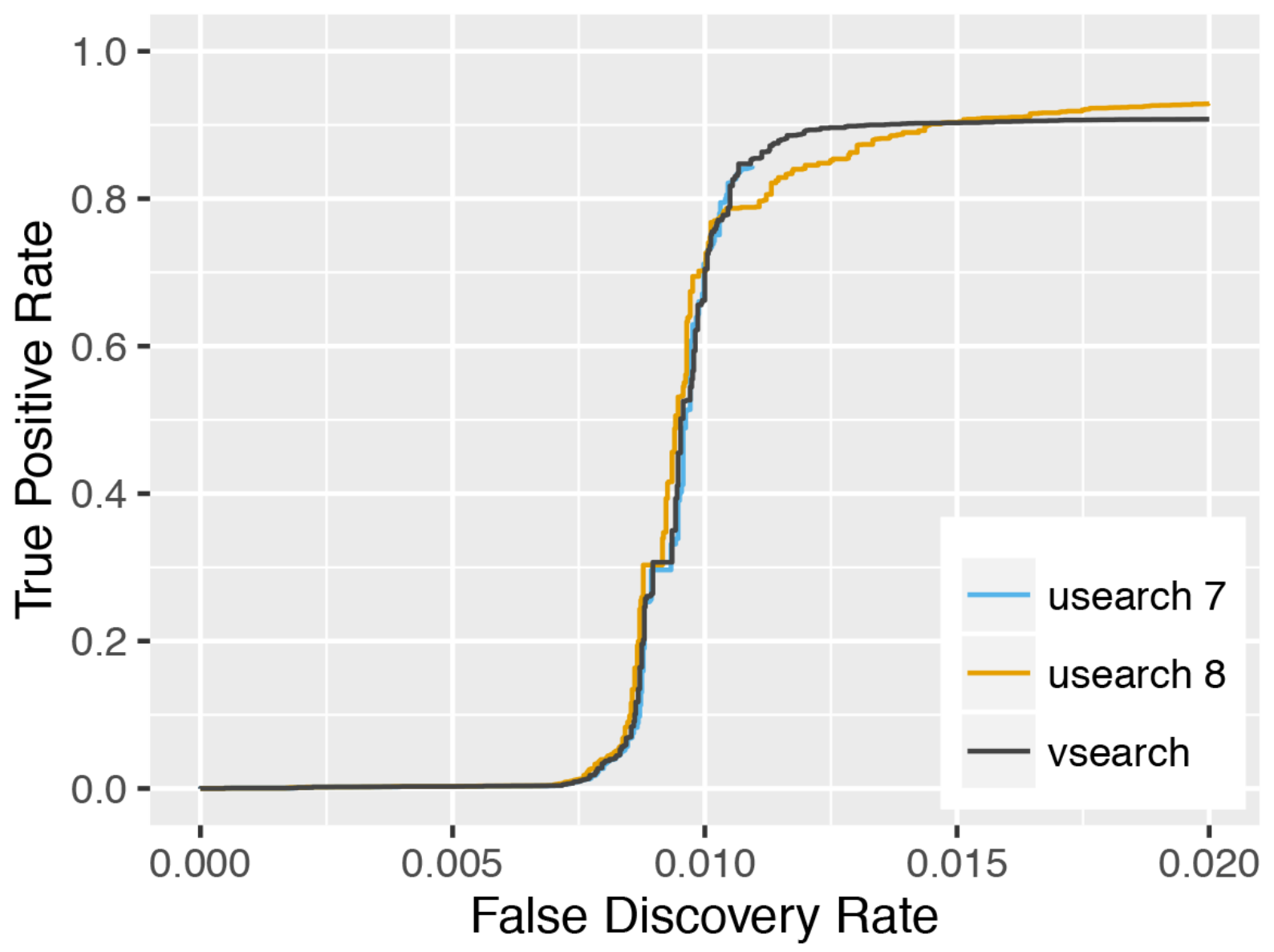

374

375

376

377

378

379

Figure 1 Search accuracy on the RFAM v11 dataset. USEARCH version 7 (blue), USEARCH version 8 (orange) and VSEARCH (black) was run using the usearch_global command on subsets of the RFAM dataset to identify members of the same families. The plot shows the true positive rate (also known as the recall or sensitivity) as a function of the false discovery rate at varying sequence similarity levels. This curve is based on data from 20 shufflings of the dataset. 


\section{Clustering}

381 Westcott and Schloss (2015) have already carried out an evaluation of the clustering

382 performance of VSEARCH. They tested the ability of several tools to assign OTUs for $16 \mathrm{~S}$

383 rRNA sequences and "demonstrated that for the greedy algorithms VSEARCH produced

384 assignments that were comparable to those produced by USEARCH making VSEARCH a viable

385 free and open source alternative to USEARCH." Schloss (2016) also evaluated de novo

386 clustering by VSEARCH.

388 We independently evaluated the clustering accuracy of USEARCH and VSEARCH as described 389 for Swarm (Mahé et al., 2014) using two mock datasets, one with an even and one with uneven 390 composition of 57 archaea and bacteria. The datasets were first dereplicated. Then the taxonomy 391 of the unique sequences was assigned by a search against the set of rRNA reference sequences 392 representing the species in the mock datasets, carried out with the usearch global command of 393 USEARCH. The sequences were shuffled randomly 10 times and clustering was performed at 20 394 different similarity levels ranging from $80 \%$ to $99 \%$ in steps of $1 \%$. Clustering was carried out in 395 two ways, first using the cluster fast command that pre-sorts the sequences by length, and then 396 using the cluster_smallmem command after first sorting the sequences by abundance using the 397 sortbysize command. We then compared the clusters obtained to the assigned species and computed the recall, precision and the adjusted Rand index of the classifications. The average values over the all shufflings are presented in Fig. 2 and Fig. 3 for the even and uneven datasets, respectively. For abundance-sorted sequences, the difference between VSEARCH and USEARCH version 8 is negligible. The difference is larger for length-sorted sequences. When using length sorting, USEARCH 8 (as well as version 7 on the even dataset) shows better precision than VSEARCH for similarity levels below $93 \%$. However, since we are comparing to species we expect the correspondence with OTUs to occur at high similarities, and in fact overall

408 The time used for clustering is shown in Fig. 4. The time used depended on the dataset, 409 algorithm and clustering threshold. The USEARCH programs were in general 2-3 times faster 410 than VSEARCH. In general the difference in speed was smaller for higher thresholds, especially 411 at $99 \%$ similarity. 


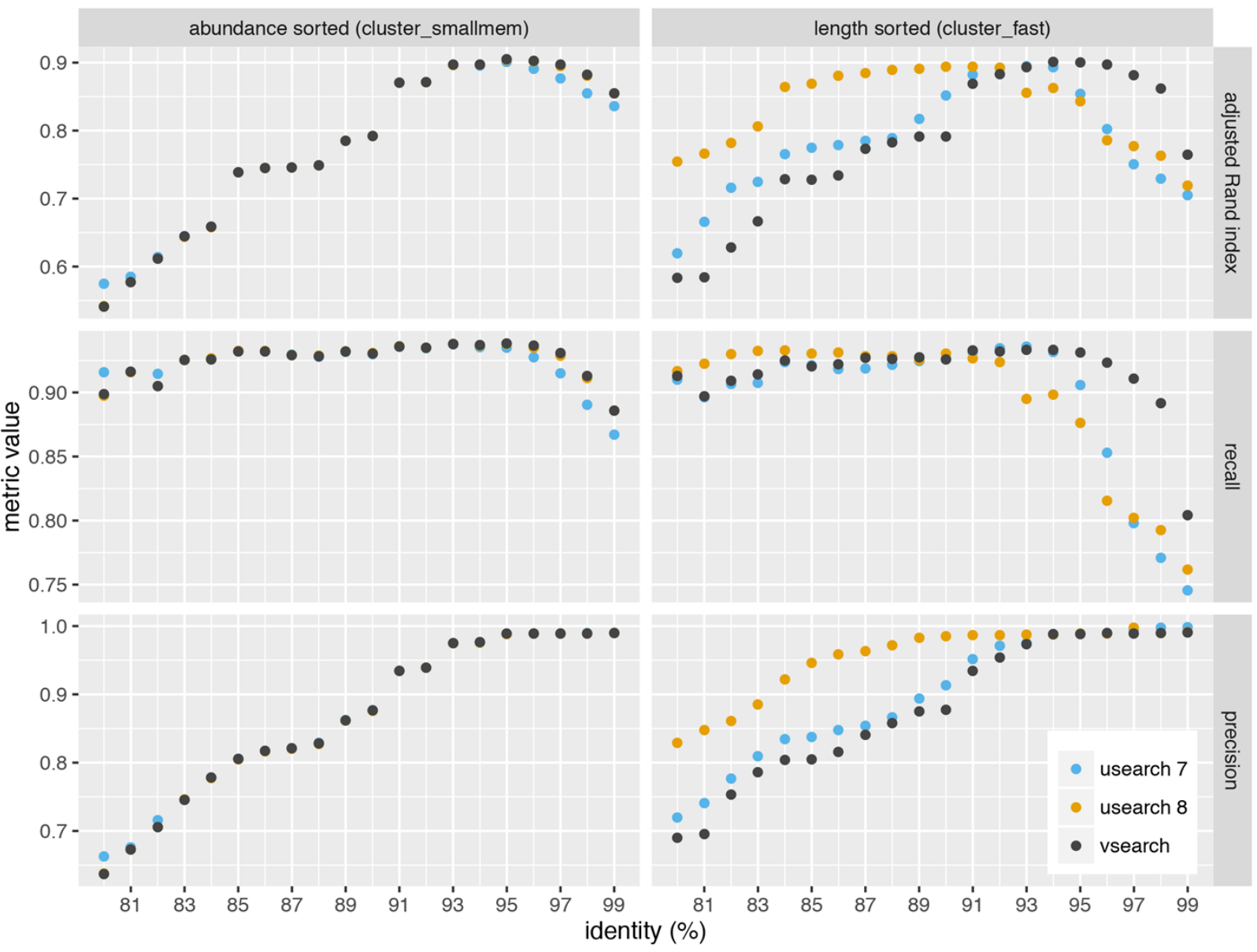

412 identity (\%)

413 Figure 2 Clustering accuracy on the even dataset. USEARCH version 7 (blue) and 8 (orange) 414 and VSEARCH (black) was run using abundance sorting (cluster_smallmem) (left) and length 415 sorting (cluster fast) (right) on the even dataset. The performance is indicated with the adjusted 416 Rand index (top), recall (middle) and precision (bottom) metrics. 


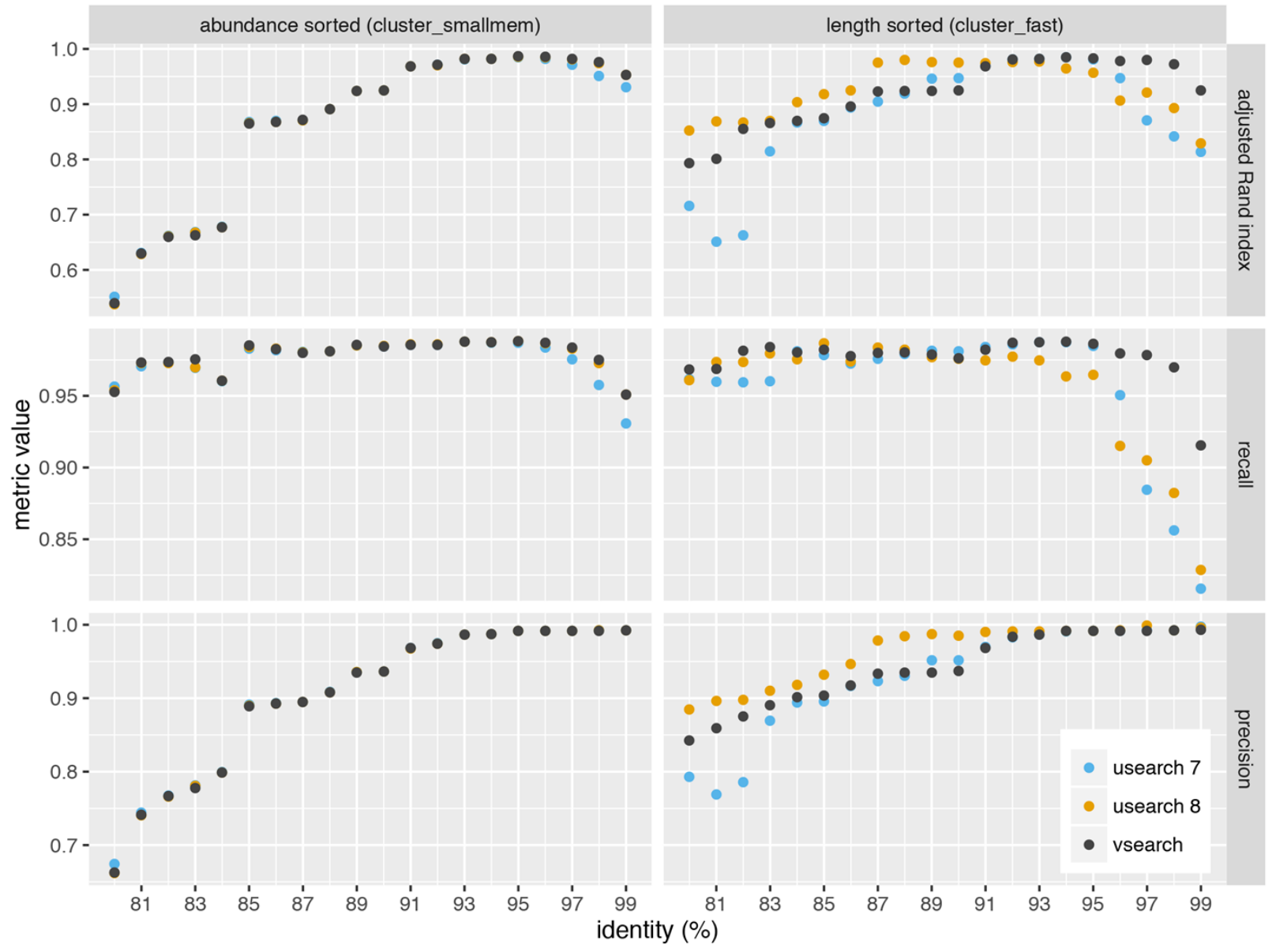

418 Figure 3 Clustering accuracy on the uneven dataset. USEARCH version 7 (blue) and 8 (orange) 419 and VSEARCH (black) was run using abundance sorting (cluster_smallmem) (left) and length 420 sorting (cluster fast) (right) on the uneven dataset. The performance is indicated with the 421 adjusted Rand index (top), recall (middle) and precision (bottom) metrics. 


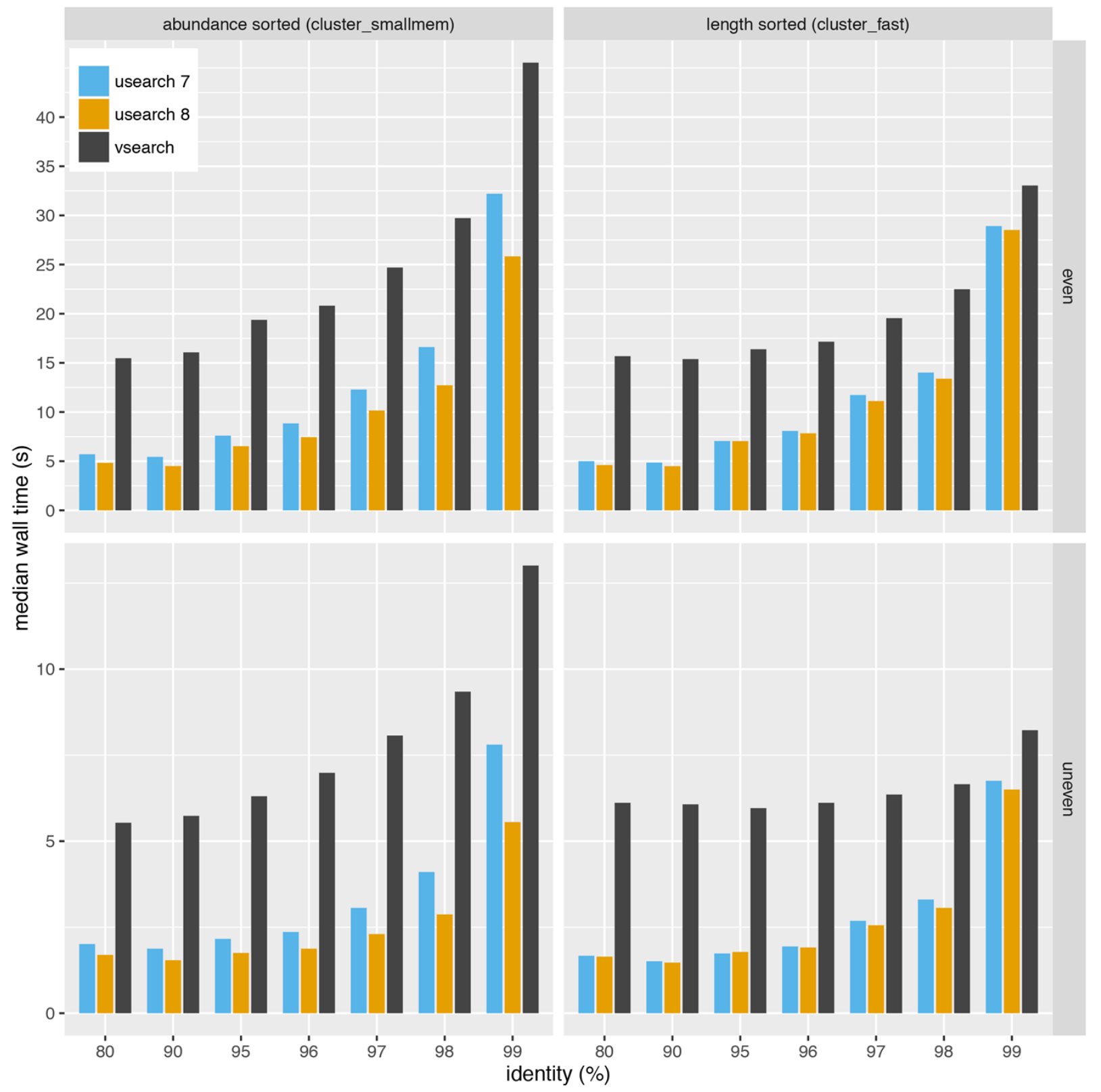

Figure 4 Clustering speed. Median wall time in seconds to cluster the even (top) and uneven 424 (bottom) datasets using USEARCH version 7 (blue) and 8 (orange) and VSEARCH (black) 425 using abundance sorting (cluster_fast) (left) and length sorting (with cluster_smallmem) (right). 


\section{Dereplication}

427 Measurements of dereplication speed were performed on the even and uneven datasets described 428 earlier as well as on the BioMarKs dataset (Karsenti et al., 2011). For full-length dereplication

429 (derep_fulllength) VSEARCH was about 40-50\% faster than USEARCH version 7 and 50-70\% 430 faster than version 8 on all three datasets. All programs were approximately equally fast on 431 prefix dereplication (derep_prefix) of the even and uneven datasets. However, prefix 432 dereplication of the BioMarKs dataset was extremely slow with USEARCH. USEARCH version 4337 used more than 4 minutes and version 8 more than 27 minutes, while VSEARCH used less 434 than 4 seconds. The prefix dereplication algorithm used in USEARCH appears ineffective when 435 dealing with short sequences. Removing the 811 sequences shorter than $200 \mathrm{bp}$ out of the 436312,503 sequences of the BioMarKs dataset reduces the running time of USEARCH version 7 437 and 8 down to just 5 and 6 seconds, respectively.

438

\section{Chimera detection}

440 We evaluated the chimera detection accuracy of VSEARCH and USEARCH in two ways, first using a method similar to that performed for UCHIME, and then using a new chimera simulation procedure from Greengenes and SILVA sequences.

443

444 First we repeated the evaluation of the uchime_ref command described in the UCHIME paper 445 (Edgar, 2011) using the SIMM dataset downloaded from http://drive5.com/uchime/uchime download.html. The dataset consists of 900 simulated chimeras that are approximately $250 \mathrm{bp}$ long. The chimeras were generated from 2, 3 or 4 segments selected randomly from 86 original sequences and have similarities in the ranges 90 $95 \%, 95-97 \%$ and $97-99 \%$ to the original sequences. They were either used unmodified or with $1-5 \%$ indels or $1-5 \%$ substitutions. We assessed the performance of i) the original open-source UCHIME version 4.2 program, ii) USEARCH version 7 , iii) USEARCH version 8 , and iv) VSEARCH. The results are shown in Table 1 and indicate that VSEARCH is superior to the other tools in almost all cases, and in particular when indels were added. The original UCHIME program was found to be quite effective, but also considerably slower than all the other tools. USEARCH was better than VSEARCH in only 3 out of 99 cases. 
456 Table 1 Chimera detection performance with the SIMM dataset. UCHIME (UC), USEARCH 457 version 7 (U7) and 8 (U8), and VSEARCH (V) was run using the uchime_ref algorithm on the 458 SIMM dataset that was originally also used to evaluate the UCHIME algorithm. Divergence is 459 the percentage of similarity to the original sequences. Noise is either zero (-) or the percentage of 460 indels (i1-i5) or substitutions (m1-5) added. The number of chimeras detected out of 100 of each 461 type is shown. The best results in each category are shaded.

462

\begin{tabular}{|c|c|c|c|c|c|c|c|c|c|c|c|c|c|}
\hline \multirow{2}{*}{\begin{tabular}{|l} 
Divergence \\
\end{tabular}} & \multirow[b]{2}{*}{ Noise } & \multicolumn{4}{|c|}{2 segments } & \multicolumn{4}{|c|}{3 segments } & \multicolumn{4}{|c|}{4 segments } \\
\hline & & $\mathbf{U C}$ & U7 & U8 & $\mathbf{V}$ & UC & U7 & U8 & $\mathbf{V}$ & UC & U7 & U8 & $\mathbf{V}$ \\
\hline \multirow[t]{11}{*}{$97-99 \%$} & - & 89 & 88 & 88 & 89 & 56 & 52 & 52 & 55 & 38 & 33 & 34 & 35 \\
\hline & i1 & 79 & 79 & 77 & 85 & 46 & 44 & 43 & 53 & 32 & 27 & 24 & 34 \\
\hline & i2 & 64 & 57 & 56 & 77 & 33 & 32 & 31 & 56 & 24 & 20 & 18 & 33 \\
\hline & i3 & 48 & 45 & 36 & 72 & 37 & 35 & 29 & 45 & 16 & 17 & 16 & 21 \\
\hline & i4 & 29 & 24 & 23 & 65 & 18 & 11 & 13 & 40 & 9 & 9 & 8 & 25 \\
\hline & i5 & 27 & 22 & 16 & 53 & 15 & 12 & 12 & 39 & 7 & 8 & 6 & 17 \\
\hline & m1 & 83 & 83 & 83 & 81 & 53 & 48 & 48 & 53 & 33 & 29 & 29 & 30 \\
\hline & $\mathbf{m} 2$ & 73 & 71 & 71 & 72 & 49 & 44 & 44 & 50 & 28 & 22 & 22 & 27 \\
\hline & m3 & 66 & 66 & 66 & 68 & 40 & 40 & 39 & 44 & 21 & 20 & 21 & 21 \\
\hline & $\mathrm{m} 4$ & 55 & 54 & 53 & 57 & 28 & 24 & 23 & 28 & 21 & 18 & 18 & 19 \\
\hline & m5 & 44 & 44 & 42 & 48 & 20 & 19 & 18 & 28 & 16 & 14 & 12 & 12 \\
\hline \multirow[t]{11}{*}{$95-97 \%$} & - & 100 & 100 & 100 & 100 & 80 & 77 & 76 & 79 & 64 & 60 & 59 & 63 \\
\hline & i1 & 100 & 98 & 98 & 100 & 77 & 75 & 72 & 75 & 54 & 55 & 53 & 61 \\
\hline & i2 & 96 & 94 & 93 & 99 & 60 & 55 & 55 & 71 & 48 & 44 & 44 & 60 \\
\hline & i3 & 86 & 82 & 82 & 95 & 61 & 50 & 52 & 70 & 38 & 36 & 31 & 53 \\
\hline & i4 & 75 & 66 & 64 & 95 & 48 & 41 & 39 & 64 & 29 & 29 & 22 & 47 \\
\hline & i5 & 64 & 58 & 53 & 86 & 37 & 32 & 25 & 60 & 24 & 19 & 19 & 46 \\
\hline & $\mathbf{m} 1$ & 99 & 99 & 99 & 99 & 76 & 73 & 73 & 76 & 60 & 57 & 57 & 60 \\
\hline & $\mathbf{m} 2$ & 98 & 97 & 97 & 97 & 71 & 69 & 69 & 71 & 50 & 48 & 46 & 48 \\
\hline & m3 & 93 & 94 & 94 & 96 & 63 & 61 & 61 & 64 & 41 & 41 & 41 & 42 \\
\hline & $\mathrm{m} 4$ & 92 & 92 & 90 & 93 & 56 & 55 & 54 & 57 & 39 & 39 & 37 & 41 \\
\hline & m5 & 86 & 86 & 85 & 86 & 53 & 51 & 51 & 56 & 35 & 35 & 34 & 34 \\
\hline \multirow[t]{11}{*}{$90-95 \%$} & - & 100 & 100 & 100 & 100 & 93 & 93 & 93 & 93 & 88 & 88 & 88 & 86 \\
\hline & i1 & 100 & 100 & 100 & 100 & 88 & 88 & 87 & 91 & 86 & 86 & 87 & 88 \\
\hline & i2 & 99 & 97 & 99 & 99 & 83 & 79 & 78 & 88 & 74 & 72 & 72 & 84 \\
\hline & i3 & 100 & 100 & 100 & 100 & 79 & 76 & 75 & 88 & 74 & 69 & 70 & 82 \\
\hline & i4 & 99 & 94 & 96 & 99 & 80 & 71 & 72 & 84 & 66 & 62 & 61 & 79 \\
\hline & i5 & 95 & 84 & 86 & 99 & 74 & 65 & 65 & 88 & 55 & 48 & 48 & 71 \\
\hline & m1 & 100 & 100 & 100 & 100 & 89 & 89 & 89 & 92 & 87 & 87 & 86 & 85 \\
\hline & m2 & 100 & 100 & 100 & 100 & 87 & 87 & 87 & 89 & 78 & 78 & 78 & 79 \\
\hline & m3 & 100 & 99 & 99 & 100 & 86 & 86 & 86 & 89 & 76 & 76 & 78 & 80 \\
\hline & $\mathrm{m} 4$ & 100 & 100 & 100 & 100 & 82 & 82 & 84 & 83 & 73 & 73 & 72 & 78 \\
\hline & $\mathrm{m5}$ & 99 & 98 & 98 & 99 & 82 & 81 & 82 & 84 & 75 & 73 & 75 & 79 \\
\hline
\end{tabular}

463 
464 Next, we tested reference-based (uchime_ref) and de novo (uchime_denovo) chimera detection 465 using sequences from the 2011 version of Greengenes downloaded from

466 http://greengenes.lbl.gov/Download/Sequence Data/Fasta data files/ (DeSantis et al., 2006) and

467 from version 106 (May 2011) of the SILVA database downloaded from https://www.arb-

468 silva.de/no_cache/download/archive/release_106/Exports/ (Quast et al., 2013). Sequences from

469 the 16S rRNA V4 region was computationally extracted using the 515F (5'-

470 GTGNCAGCMGCCGCGGTAA-3') and 806R (5'-GGACTACHVGGGTWTCTAAT-3')

471 primers, and 8,000 reads were randomly selected from each database. PCR was simulated using

472 a new simulation algorithm known as Simera (Nichols and Quince, 2016) (available at

473 https://github.com/bnichols1979/Simera) that includes amplification and creation of PCR

474 artefacts like chimeras. We sampled 30,000 reads (-s 30000) and generated 20,000 potential

475 chimeras (-c 20000). Defaults were used for other options to Simera. The output sequences were

476 then fed into an Illumina MiSeq noise simulator (Schirmer et al., 2015) ending up with 14,966

477 reads based on Greengenes and 14,952 reads based on SILVA, of which 1,262 and 1,640 reads

478 contain chimeric sequences, respectively. Next, the sequences were either clustered using the

479 cluster fast command at 97\% identity or dereplicated. VSEARCH and USEARCH version 7 and

4808 were run using the uchime_denovo command and then using the uchime_ref command with the

481 Gold database downloaded from http://drive5.com/uchime/uchime download.html as the

482 reference database. To assess the performance, the results were sorted based on the chimera

483 score, and then the ability to classify individual sequences correctly into chimeric and non-

484 chimeric was plotted as ROC curves. The curves reflect the accuracy of classifying individual

485 reads, not clusters, as abundances were taken into account. The plots in Fig. 5 and Fig. 6 show

486 that de novo chimera detection performs better than reference-based detection, with the SILVA

487 dataset in particular, but it does of course depend on the reference database used. VSEARCH

488 performs better than both versions of USEARCH for de novo chimera detection. For reference-

489 based detection VSEARCH also performs better for the Greengenes dataset, while none of the

490 programs works well with the SILVA dataset. Clustering at 97\% appears to be more appropriate

491 than dereplication. In this test, the USEARCH programs were about twice as fast as VSEARCH

492 for de novo detection, while they were about 10-30\% faster than VSEARCH for reference-based

493 detection. 


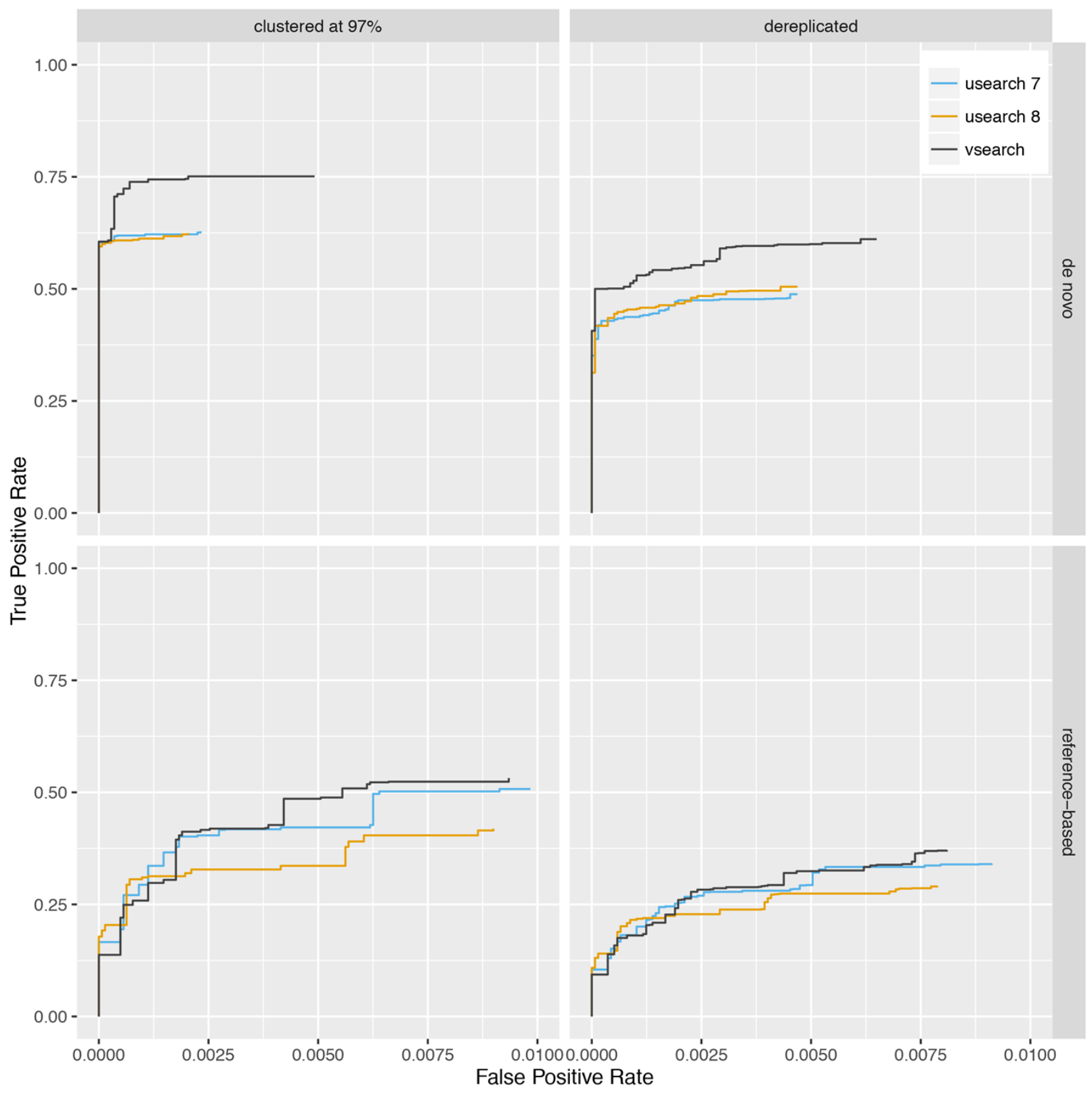

496 Figure 5 Chimera detection performance with the Greengenes dataset shown with ROC curves.

497 USEARCH version 7 (blue) and 8 (orange) and VSEARCH (black) was run using the 498 uchime_denovo (top) and the uchime_ref(bottom) commands on simulated Illumina data based 499 on the Greengenes database that has either been clustered with a 97\% identity threshold (using 500 the cluster fast command in VSEARCH) (left) or dereplicated (using the derep_fullength 501 command in VSEARCH) (right). 


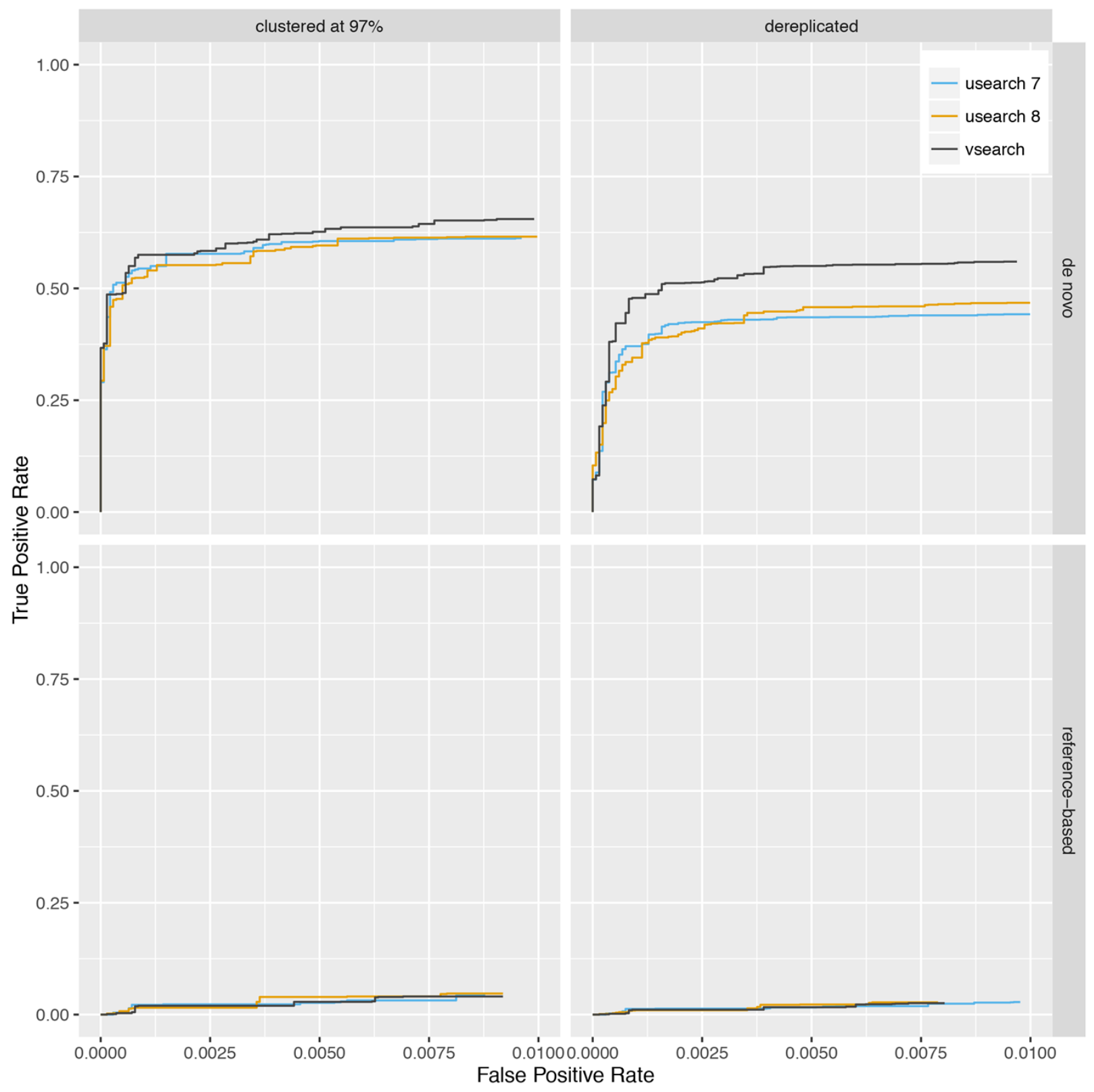

504 Figure 6 Chimera detection performance on the SILVA dataset shown with ROC curves.

505 USEARCH version 7 (blue) and 8 (orange) and VSEARCH (black) was run using the 506 uchime_denovo (top) and the uchime_ref(bottom) commands on simulated Illumina data based 507 on the SILVA database that has either been clustered with a 97\% identity threshold (using the 508 cluster_fast command in USEARCH) (left) or dereplicated (using the derep_fullength command 509 in VSEARCH) (right). 


\section{Merging of paired-end reads}

511 Evaluation of paired-end reads merging performance was carried out in a manner similar to that

512 described for the evaluation of PEAR (Zhang et al., 2014). We used whole genome sequencing

513 data from Staphylococcus aureus subspecies aureus strain USA 300 TCH 1516 sequenced by

514 MacCallum et al. (2009) and retrieved from the GAGE-B repository (http://ccb.jhu.edu/gage b/).

515 The S.aureus reads were $101 \mathrm{bp}$ long from on average $180 \mathrm{bp}$ long fragments, giving a 45X

516 coverage of the genome. We also used Methylococcus capsulatus strain Bath 16S rRNA V3

517 region amplicon reads sequenced by Masella et al. (2012). These reads were $108 \mathrm{bp}$ long and the

518 pairs should have an overlap of exactly $18 \mathrm{bp}$. Merging options were set to allow a minimum

519 overlap of $10 \mathrm{bp}$ and a maximum of 5 mismatches (USEARCH 7 and 8 have different default

520 values for those), while other options were left at defaults. All programs were run with 8 threads.

521 Merged sequences that could be perfectly aligned to their respective reference sequences (either

522 the entire genome or the specific rRNA region) using BWA MEM (Li et al., 2009) were

523 considered correctly merged. The results are shown in Table 2 . The numbers indicate that

524 USEARCH version 7 merges the most reads for both bacteria, but also has the lowest percentage

525 of correctly merged pairs of those merged. USEARCH version 8 merges the fewest reads, but

526 has the highest percentage of correctly merged reads of those merged. VSEARCH is in the

527 middle by merging more reads than USEARCH 8 with only a small decrease in the percentage of

528 correct merges. VSEARCH is about twice as fast as USEARCH 8 and 4-5 times faster than

529 USEARCH version 7.

530 
531 Table 2. Paired-end reads merging performance. The number of sequence pairs, merged pairs, 532 and correctly merged pairs are shown for each bacterium and program. The percentage of reads 533 merged, as well as the percentage of correctly merged reads both of the merged reads and of all 534 reads are also shown. Times are in seconds using 8 threads.

535

\begin{tabular}{|l|l|r|r|r|r|r|r|r|}
\hline Bacterium & Program & Pairs & Merged & Correct & \%Merged & \%Cor/Mer & \%Cor/All & Time (s) \\
\hline \multirow{4}{*}{ Staphylococcus aureus } & USEARCH 7 & 647,052 & 273,438 & 270,849 & 42.26 & 99.05 & 41.86 & 11.65 \\
\cline { 2 - 9 } & USEARCH 8 & 647,052 & 203,729 & 202,003 & 31.49 & 99.15 & 31.22 & 4.69 \\
\cline { 2 - 9 } & VSEARCH & 647,052 & 214,988 & 213,103 & 33.23 & 99.12 & 32.93 & 2.15 \\
\hline \multirow{2}{*}{$\begin{array}{l}\text { Methylococcus capsulatus } \\
\text { strain Bath }\end{array}$} & USEARCH 7 & 673,845 & 643,903 & 642,720 & 95.56 & 99.82 & 95.38 & 14.43 \\
\cline { 2 - 9 } & USEARCH 8 & 673,845 & 554,099 & 553,747 & 82.23 & 99.94 & 82.18 & 6.27 \\
\cline { 2 - 9 } & VSEARCH & 673,845 & 581,752 & 581,346 & 86.33 & 99.93 & 86.27 & 3.61 \\
\hline
\end{tabular}

536 


\section{Subsampling}

538 We evaluated the subsampling commands of USEARCH version 8 and VSEARCH to check if 539 the results obtained correspond to those expected. We performed 10,000 random subsamplings 540 of $5 \%$ of the 9.5 million unique sequences in the TARA V9 dataset (Karsenti et al., 2011). To 541 make this possible with the 32-bit USEARCH, we first downsampled the dataset once to $10 \%$ 542 using VSEARCH and then randomly subsampled it again at $50 \%$ with either USEARCH or 543 VSEARCH. Plots of the distribution of the abundance of the most abundant sequence in each 544 subsampling are shown in Fig. 7. The highest amplicon abundance in the original dataset is $54515,638,316$. After the initial 10\% subsampling, the highest abundance was 1,564,267. After the 546 second subsampling, the top abundances should therefore have a distribution centred on a value 547 of 782,133.5. As can be seen from the figure, the USEARCH distribution has a mean that is 548 about 2,000 too small, while the VSEARCH distribution is correctly centred on the expected 549 value. Subsampling experiments were also performed at $2.5 \%, 1.5 \%$ and $0.5 \%$ with similar 550 results, although the errors were of decreasing size. USEARCH seems to under-sample abundant 551 amplicons and to over-sample rare amplicons. 


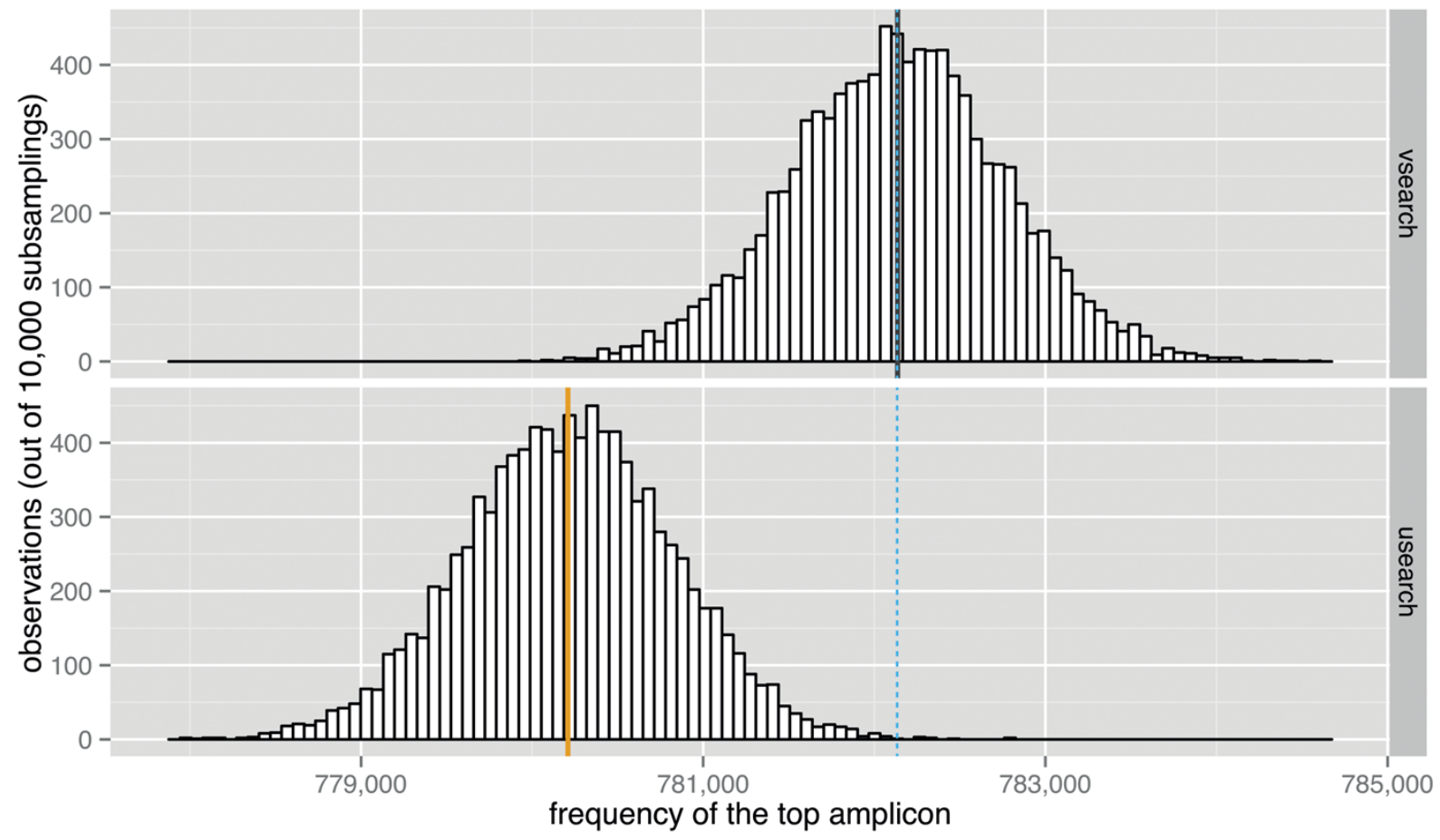

553 Figure 7 Subsampling performance. The observed distribution of the maximum amplicon 554 abundance in 10,000 random subsamplings of 5\% of the TARA V9 dataset results using 555 VSEARCH (top, black) and USEARCH version 8 (bottom, orange) is shown. The expected 556 mean abundance is 782,133.5 (blue dashed line). 


\section{Conclusions}

558 VSEARCH supports almost all of the commands and options for nucleotide sequence analysis in

559 USEARCH version 7 as well several new features. It has a 64-bit design and handles large

560 datasets virtually only limited by the amount of available memory. We have demonstrated that

561 VSEARCH is in general more accurate than USEARCH when performing searching, clustering,

562 chimera detection and subsampling. The accuracy is on a par with USEARCH for paired-end

563 reads merging. VSEARCH is faster than USEARCH when performing dereplication and

564 merging of paired-end reads, but slower for clustering and chimera detection. We will continue

565 to improve the accuracy, speed and robustness of VSEARCH in the future, as well as adding new

566 features.

567

568

Availability

569 VSEARCH is freely available at https://github.com/torognes/vsearch under a dual license, either

570 the GNU General Public License version 3, or the BSD 2-clause license. Binaries are provided

571 for x86-64 systems running GNU/Linux or OS X (10.7 or higher).

572

573

574

Thanks to the work of several people, there is now a vsearch package in Debian and a vsearch package for Homebrew, as well as a Galaxy wrapper for VSEARCH in the Galaxy ToolShed.

575

576

577

578

579

580

581

582

583

584

585

586

587

588

589

590

591

592

593

594

\section{Acknowledgements}

We highly appreciate the feedback from numerous people who submitted bug reports and suggestions for features.

Thanks to Melanie Schirmer for noise generation on sequences for chimera detection.

\section{References}

Altschul SF, Gish W, Miller W, Myers EW, Lipman DJ. 1990. Basic local alignment search tool. Journal of Molecular Biology, 215:403-410. DOI: 10.1016/S0022-2836(05)80360-2

Burge SW, Daub J, Eberhardt R, Tate J, Barquist L, Nawrocki EP, Eddy SR, Gardner PP, Bateman A. 2013. Rfam 11.0: 10 years of RNA families. Nucleic Acids Research, 41(D1):D226D232. DOI: $10.1093 /$ nar/gks 1005

Caporaso JG, Kuczynski J, Stombaugh J, Bittinger K, Bushman FD, Costello EK, Fierer N, Peña AG, Goodrich JK, Gordon JI, Huttley GA, Kelley ST, Knights D, Koenig JE, Ley RE, Lozupone CA, McDonald D, Muegge BD, Pirrung M, Reeder J, Sevinsky JR, Turnbaugh PJ, Walters WA, Widmann J, Yatsunenko T, Zaneveld J, Knight R. 2010. QIIME allows analysis of highthroughput community sequencing data. Nature Methods, 7:335-336. DOI: 10.1038/nmeth.f.303 
596 Cock PJA, Fields CJ, Goto N, Heuer ML and Rice PM. 2010. The Sanger FASTQ file format for

597 sequences with quality scores, and the Solexa/Illumina FASTQ variants. Nucleic Acids

598 Research, 38(6):1767-1771. DOI: 10.1093/nar/gkp1137

599

600 DeSantis TZ, Hugenholtz P, Larsen N, Rojas M, Brodie EL, Keller K, Huber T, Dalevi D, Hu P, 601 Andersen GL. 2006. Greengenes, a Chimera-Checked 16S rRNA Gene Database and Workbench 602 Compatible with ARB. Applied and Environmental Microbiology, 72(7):5069-72. DOI:

603 10.1128/AEM.03006-05

604

605 Eastlake D, Jones P. 2001. US Secure Hash Algorithm 1 (SHA). Internet RFC 3174. Available at $606 \mathrm{ftp}: / / \mathrm{ftp} . \mathrm{rfc}-\mathrm{editor} . \mathrm{org} /$ in-notes/rfc3174.txt

607

608

609

Edgar RC. 2010. Search and clustering orders of magnitude faster than BLAST. Bioinformatics, 26(19):2460-2461. DOI: 10.1093/bioinformatics/btq461

610

611 Edgar RC. 2013. UPARSE: highly accurate OTU sequences from microbial amplicon reads.

612 Nature Methods, 10(10):996-8. DOI: 10.1038/nmeth.2604

613

614 Edgar RC, Flyvbjerg H. 2015. Error filtering, pair assembly and error correction for next-

615 generation sequencing reads. Bioinformatics, 31(21):3476-3482. DOI:

616 10.1093/bioinformatics/btv40

617

618 Edgar RC, Haas BJ, Clemente JC, Quince C, Knight R. 2011. UCHIME improves sensitivity and

619 speed of chimera detection. Bioinformatics, 27(16):2194-2200. DOI:

620 10.1093/bioinformatics/btr381

621

622

Fowler G, Noll LC, Vo P. 1991. Fowler / Noll / Vo (FNV) hash. Available at

623

http://www.isthe.com/chongo/tech/comp/fnv/index.html

624

625

Gailly JL, Adler M. 2016. zlib: A Massively Spiffy Yet Delicately Unobtrusive Compression

626 Library. Available at http://www.zlib.net/ (accessed 3 August 2016)

627

628

Gilbert JA, Jansson JK, Knight R. 2014. The Earth Microbiome project: successes and

629 aspirations. BMC Biology, 12:69. DOI: 10.1186/s12915-014-0069-1

630

631 Guillou L, Bachar D, Audic S, Bass D, Berney C, Bittner L, Boutte C, Burgaud G, de Vargas C, 632 Decelle J, del Campo J, Dolan J, Dunthorn M, Edvardsen B, Holzmann M, Kooistra W, Lara E, 633 Lebescot N, Logares R, Mahé F, Massana R, Montresor M, Morard R, Not F, Pawlowski J,

634 Probert I, Sauvadet A.-L, Siano R, Stoeck T, Vaulot D, Zimmermann P, Christen R. 2013. The 
635 Protist Ribosomal Reference database (PR2): a catalog of unicellular eukaryote Small Sub-Unit

636 rRNA sequences with curated taxonomy. Nucleic Acids Research, 41(D1):D597-D604. DOI:

637 10.1093/nar/gks1160

638

639 He Y, Caporaso JG, Jiang XT, Sheng HF, Huse SM, Rideout JR, Edgar RC, Kopylova E, 640 Walters WA, Knight R and Zhou HW. 2015. Stability of operational taxonomic units: an 641 important but neglected property for analyzing microbial diversity. Microbiome, 3:20. DOI:

642 10.1186/s40168-015-0081-x

643

644 Hirschberg DS. 1975. A linear space algorithm for computing maximal common subsequences. 645 Communications of the ACM, 18(6):341-343. DOI: $10.1145 / 360825.360861$

646

647

648

Human Microbiome Project Consortium. 2012. Structure, function and diversity of the healthy

649

650 human microbiome. Nature, 486:207-214. DOI: 10.1038/nature11234

Karsenti E, González Acinas S, Bork P, Bowler C, de Vargas C, Raes J, Sullivan M. B, Arendt

651 D, Benzoni F, Claverie J.-M, Follows M, Jaillon O, Gorsky G, Hingamp P, Iudicone D, KandelsVelayoudon D, Weissenbach J, Wincker P, the Tara Oceans Consortium. 2011. A holistic approach to marine eco-systems biology. PLoS Biology, 9(10):e1001177. DOI: 10.1371/journal.pbio.1001177

656

Kopylova E, Navas-Molina JA, Mercier C, Xu ZZ, Mahé F, He Y, Zhou HW, Rognes T,

658 Caporaso JG, Knight R. 2016. Open-Source Sequence Clustering Methods Improve the State Of the Art. mSystems, 1(1):e00003-15. DOI: 10.1128/mSystems.00003-15

660

Li H, Durbin R. 2009. Fast and accurate short read alignment with Burrows-Wheeler transform.

662 Bioinformatics, 25(14):1754-60. DOI: 10.1093/bioinformatics/btp324

663

664

Logares R, Audic S, Bass D, Bittner L, Boutte C, Christen R, Claverie J.-M, Decelle J, Dolan J.

665 R, Dunthorn M, Edvardsen B, Gobet A, Kooistra W. H. C. F, Mahé F, Not F, Ogata H, Pawlowski J, Pernice M. C, Romac S, Shalchian-Tabrizi K, Simon N, Stoeck T, Santini S, Siano R, Wincker P, Zingone A, Richards T, de Vargas C, Massana R. 2014. The patterning of rare and abundant community assemblages in coastal marine-planktonic microbial eukaryotes. Current Biology, 24(8):813-821. DOI: 10.1016/j.cub.2014.02.050

670

671

672

Mahé F, Rognes T, Quince C, de Vargas C, Dunthorn M. 2014. Swarm: robust and fast

673 
674 Masella AP, Bartram AK, Truszkowski JM, Brown DG and Neufeld JD. 2012. PANDAseq:

675 paired-end assembler for illumina sequences. BMC Bioinformatics, 13:31. DOI: 10.1186/1471-

$676 \quad 2105-13-31$

677

678 Myers EW, Miller W. 1988. Optimal alignments in linear space. Computer Applications in the 679 Biosciences, 4(1):11-17. DOI: 10.1093/bioinformatics/4.1.11

680

681 Needleman SB, Wunsch CD. 1970. A general method applicable to the search for similarities in 682 the amino acid sequence of two proteins. Journal of Molecular Biology, 48(3):443-53. DOI:

683 10.1016/0022-2836(70)90057-4.

684

685 Nichols B, Quince C. 2016. Simera: Modelling the PCR Process to Simulate Realistic Chimera 686 Formation. bioRxiv, 072447. DOI: 10.1101/072447

687

688 Quast C, Pruesse E, Yilmaz P, Gerken J, Schweer T, Yarza P, Peplies J, Glöckner FO. 2013. The 689 SILVA ribosomal RNA gene database project: improved data processing and web-based tools.

690 Nucleic Acids Research, 41(D1):D590-D596. DOI: 10.1093/nar/gks1219

691

692

Rivest R. 1992. The MD5 Message-Digest Algorithm. Internet RFC 1321. Available at

693

694

695 ftp://ftp.rfc-editor.org/in-notes/rfc1321.txt

Rockström J, Steffen W, Noone K, Persson A, Chapin FS 3rd, Lambin EF, Lenton TM, Scheffer

697 M, Folke C, Schellnhuber HJ, Nykvist B, de Wit CA, Hughes T, van der Leeuw S, Rodhe H, Sörlin S, Snyder PK, Costanza R, Svedin U, Falkenmark M, Karlberg L, Corell RW, Fabry VJ,

699 Hansen J, Walker B, Liverman D, Richardson K, Crutzen P, Foley JA. 2009. A safe operating space for humanity. Nature, 461(7263):472-5. DOI: 10.1038/461472a

700

701

702

703

704

705

Rognes T. 2011. Faster Smith-Waterman database searches by inter-sequence SIMD parallelisation. BMC Bioinformatics, 12:221. DOI: 10.1186/1471-2105-12-221

Schirmer M, Ijaz UZ, D'Amore R, Hall N, Sloan WT, Quince C. 2015. Insight into biases and sequencing errors for amplicon sequencing with the Illumina MiSeq platform. Nucleic Acids Research, 43(6):e37. doi: 10.1093/nar/gku1341

Schloss PD (2016) Application of a Database-Independent Approach To Assess the Quality of

710 Operational Taxonomic Unit Picking Methods. mSystems, 1(2):e00027-16. DOI:

711

712 Schloss PD, Westcott SL, Ryabin T, Hall JR, Hartmann M, Hollister EB, Lesniewski RA, 713 Oakley BB, Parks DH, Robinson CJ, Sahl JW, Stres B, Thallinger GG, Van Horn DJ, Weber CF. 
714 2009. Introducing mothur: open-source, platform-independent, community-supported software

715 for describing and comparing microbial communities. Applied and Environmental Microbiology,

716 75:7537-7541. DOI: 10.1128/AEM.01541-09.

717

718 Seward J. 2016. bzip2 and libbzip2. Available at http://www.bzip.org/ (accessed 3 August 2016)

719

720

Song K, Ren J, Reinert G, Deng M, Waterman MS, Sun F. 2014. New developments of

721

alignment-free sequence comparison: measures, statistics and next-generation sequencing.

722

Briefings in Bioinformatics, 15(3):343-53. DOI: 10.1093/bib/bbt067

723

724 Steffen W, Richardson K, Rockström J, Cornell SE, Fetzer I, Bennett EM, Biggs R, Carpenter

725 SR, de Vries W, de Wit CA, Folke C, Gerten D, Heinke J, Mace GM, Persson LM, Ramanathan

726 V, Reyers B, Sörlin S. 2015. Sustainability. Planetary boundaries: guiding human development

727

728

729

Westcott SL, Schloss PD. 2015. De novo clustering methods outperform reference-based

730 methods for assigning 16S rRNA gene sequences to operational taxonomic units. PeerJ, 3:e1487

731

DOI: 10.7717 peerj. 1487

732

733

Zhang J, Kobert K, Flouri T, Stamatakis A. 2014. PEAR: a fast and accurate Illumina Paired-End

734 reAd mergeR. Bioinformatics, 30(5):614-20. DOI: 10.1093/bioinformatics/btt593

735

736 Declarations

737 Competing Interests

738 The authors declare there are no competing interests.

739

740 Funding statement

741 This research was supported in part with computational resources at the University of Oslo

742 provided by NOTUR project NN9383K and funded by the Research Council of Norway.

743

744

BN was funded by BBSRC CASE studentship supported by Unilever.

745

746 CQ was funded through the MRC Cloud Infrastructure for Microbial Bioinformatics (CLIMB)

747

748

749 project (MR/L015\-080/1) through fellowship (MR/M50161X/1).

750

FM was supported by the Deutsche Forschungsgemeinschaft (grant \#DU1319/1-1). 


\section{Author contributions}

752 Which authors conceived and designed the experiments? TR, TF, BN, CQ, FM

753 Which authors performed the experiments? TR, TF, BN, FM

754 Which authors analyzed the data? TR, TF, BN, CQ, FM

755 Which authors contributed reagents/materials/analysis tools? TR, TF, BN, CQ, FM

756 Which authors wrote the manuscript? TR, TF, BN, CQ, FM

757 Which authors prepared the figures and/or tables? TR, BN, FM

758 Which authors reviewed drafts of the paper? TR, TF, BN, CQ, FM

759 Which authors made other contributions? None 